\title{
Percutaneouse endoscopic gastrostomy tube outcomes are heavily influenced by indication and concurrent illness
}

\author{
S. A. Sawhney, D. Barber and A. W. McKinlay \\ McKinlay, Aberdeen Royal Infirmary, Ashgrove House, Foresterhill, AB25 2ZA, Scotland, UK
}

Feeding PEG tubes are indicated for long-term ( $>30 \mathrm{~d}$ ) nutrition in patients with mechanical or neurological feeding difficulty. Although it is a minor procedure, insertion is invasive and associated with complications. A recent NCEPOD audit raised concerns of early deaths $(<30 \mathrm{~d})$ following insertions and frequent futility ${ }^{(1)}$. Furthermore, quality-of-life studies have failed to demonstrate significant benefit.

Primary PEG insertions for feeding in Aberdeen Royal Infirmary over 2007 were subdivided by indication and followed up by intention to treat over $180 \mathrm{~d}$. Venting tubes and revision procedures were excluded.

Hundred-and-two primary PEG insertions occurred in 2007. Seven, 30 and $180 \mathrm{~d}$ mortality rates were $4(3.9 \%), 19(18.6 \%)$ and 43 $(42.1 \%)$, respectively. Thirty-one $(30.4 \%)$ patients died without being discharged from hospital. There were significant associations between pre-procedure CRP $(P=0.032)$ and serum albumin $(P<0.001)$ and subsequent mortality. Patient outcomes differed markedly depending on the primary PEG indication. A large proportion of the dementia patients died early following the procedure. Half of the stroke patients died within 6 months and a similar proportion died during the same admission as the procedure.

\begin{tabular}{|c|c|c|c|c|c|c|}
\hline Indication & Stroke & Neurosurgical & ENT & GI/Surgical/Nutrition & Neurodegenerative & Dementia \\
\hline$N$ & 32 & 13 & 17 & 14 & 14 & 7 \\
\hline Age (median) & 80.8 & 62.0 & 67.4 & 60.0 & 68.4 & 82.0 \\
\hline Death by $7 d$ & $1(3.1 \%)$ & 0 & $3(17.6 \%)$ & 0 & 0 & 0 \\
\hline Death by $30 \mathrm{~d}$ & $6(18.8 \%)$ & $3(23.1 \%)$ & $5(29.4 \%)$ & 0 & $1(7.1 \%)$ & $4(57.1 \%)$ \\
\hline Death by $180 \mathrm{~d}$ & $16(50 \%)$ & $3(23.1 \%)$ & $10(58.8 \%)$ & $4(28.6 \%)$ & $5(35.7 \%)$ & $5(71.4 \%)$ \\
\hline Death before discharge & $14(43.8 \%)$ & $3(23.1 \%)$ & $7(42.2 \%)$ & $3(21.4 \%)$ & $2(14.3 \%)$ & $2(28.6 \%)$ \\
\hline
\end{tabular}

Prognosis following PEG insertion varies widely with underlying condition. CRP and Albumin, as markers of either concurrent illness or disease burden, also predict a poorer outcome. These factors are important both when evaluating suitability for PEG insertion and when communicating prognosis with patients and families if a PEG is being considered. While it is methodologically challenging to assess survival benefit, the poor prognosis in stroke patients with PEG tubes suggests that their use should be considered on a case-by-case basis only and primarily with palliative intent where quality of life may benefit. It is difficult to justify PEG tube use in dementia on these results.

1. Johnston SD, Tham TCK \& Mason M (2008) Death after PEG: results of the National Confidential Enquiry into Patient Outcome and Death. Gastrointest Endosc 68(2), 223-227. 